\title{
A Simulation Analysis of Large Scale Path Loss in an Underwater Acoustic Network
}

\author{
Jesús Llor \\ Miguel Hernández University \\ Avenida de la Universidad $\mathrm{s} / \mathrm{n}$, \\ Edificio Alcudia \\ 03202, Elche (Alicante) Spain
}

\author{
Milica Stojanovic \\ Northeastern University \\ 360 Huntington Avenue \\ Boston, MA \\ 02115 USA
}

\author{
Manuel P. Malumbres \\ Miguel Hernández University \\ Avenida de la Universidad s/n, \\ Edificio Alcudia \\ 03202, Elche (Alicante) Spain
}

\begin{abstract}
Propagation conditions in an underwater acoustic channel are known to vary in time, causing the received signal strength to deviate from the nominal value predicted by a deterministic propagation model. To facilitate large-scale system design in such conditions (e.g. power allocation), we develop a statistical propagation model in which the transmission loss is treated as a random variable. By repetitive computation of acoustic field using ray tracing for a set of varying environmental conditions (surface height, wave activity, small displacements of transmitter and receiver around nominal locations), an ensemble of transmission losses is compiled which is then used to infer the statistical model parameters. A reasonable agreement is found with log-normal distribution, whose mean obeys a log-distance increases, and whose variance appears to be constant for a certain range of inter-node distances in a given deployment location. The statistical model is deemed useful for higher-level system planning, where simulation is needed to assess the performance of candidate network protocols under various resource allocation policies, i.e. to determine the transmit power and bandwidth allocation necessary to achieve a desired level of performance (connectivity, throughput, reliability, etc.).
\end{abstract}

\section{INTRODUCTION}

The growing need for ocean observation and remote sensing has recently motivated a surge in research publications as well as several experimental efforts (e.g. [1]) in the area of underwater acoustic networks. Crucial to these developments is the understanding of propagation conditions that define the time-varying and location-sensitive acoustic environment, not only from the viewpoint of small-scale, rapid signal fluctuations that affect the performance of the physical layer techniques, but also from the viewpoint of large-scale, slow fluctuations of the received signal power that affect the performance of higher network layers. This fact has been gaining recognition in the research community, leading to an increased awareness about the need for network simulators that take into account the physics of acoustic propagation [1][4]. As a result, the first publicly available acoustic network simulators have emerged [2], and more are likely to come.

One of the challenges in the design of underwater acoustic networks is the allocation of power across different network nodes. This task is exacerbated by the spatial and temporal variation of the large-scale transmission loss, and the lack of statistical models that capture these apparently random phenomena.

While it is well known from field experiments that the received power varies in time around the nominal value predicted by a deterministic propagation model, little is known about the statistical nature of these variations. Literature on this topic is scarce; however, several recent references indicate that the received signal strength obeys a log-normal distribution (e.g. [5][6]). A good system design has to budget for signal strength variations in order to ensure a desired level of network performance (e.g. connectivity), and the budgeting task can be made much easier if the statistics of the underlying process are known.

In this paper, we analyze those random variations in the large-scale transmission loss that are mainly governed by the environmental factors such as surface activity (waves) for a particular network scenario. We begin by employing a prediction model based on the Bellhop ray tracing tool [7]. Such a deterministic model provides accurate results for a specific geometry of the system, but does not reflect the changes that occur as the geometry changes slightly due to either surface motion or transmitter/receiver motion. Fig.1 illustrates this situation for a point-to-point link. It shows an ensemble of transmission losses calculated by the Bellhop model for a set of varying surface conditions, each slightly different from the nominal.

While it is possible in principle to run a deterministic propagation model for a large number of different surface conditions, the underlying computational demands are high. In a large network, it is ineffective, and possibly not even feasible, to run a complex prediction model for each packet transmission. A statistical prediction model then becomes necessary.

The goal of our work is to employ an existing deterministic prediction model (DPM) such as the ray tracer [7] to generate an ensemble of channel responses corresponding to varying propagation conditions in a given network. Using the soobtained values, we then conduct a statistical analysis to obtain the probability density function (pdf) of the large-scale transmission loss. The result is a statistical prediction model (SPM) that is easy to employ for network design and analysis.

The rest of this paper is organized as follows. In Sec.II we outline a specific system example, and discuss the computational demands of deterministic propagation modeling. In Sec.III, we present the results of deterministic modeling and develop an underlying statistical model. In Sec.IV we discuss the implications that statistical modeling can have on network simulation, and we conclude in Sec.V. 


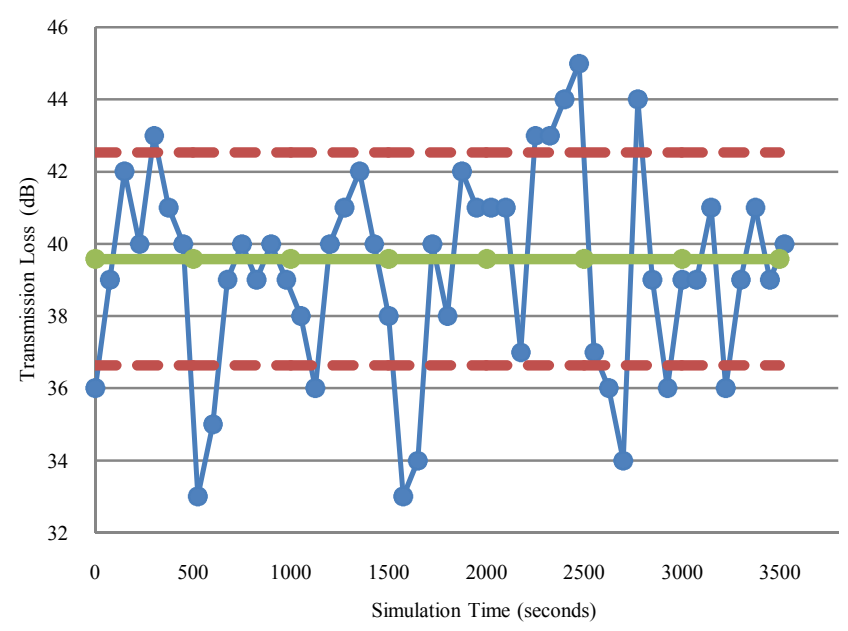

Figure 1. An ensemble of transmission losses calculated by the Bellhop model. Solid line indicates the average calculated over the total run time. Dashed lines indicate the values of one standard deviation $\sigma$.

\section{SYSTEM SET-UP}

The network of interest is located in coastal waters near Valencia, Spain, at coordinates $39^{\circ} 48^{\prime} 13.14^{\prime \prime} \mathrm{N}$ and $0^{\circ} 4$ '34.53"W. It consists of eight nodes arranged in a linear topology, as illustrated in Fig.2.

In general, an arbitrary network location can be considered, for which the bathymetry, floor sediment and sound speed profile can be found in the online databases [8], [9], [10]. In our example, the source is assumed to be at one end, and the rest of the nodes are placed at different distances ranging from $500 \mathrm{~m}$ to $3,700 \mathrm{~m}$. The nodes are at a depth of 10 meters, while the water depth varies from $25 \mathrm{~m}$ to $35 \mathrm{~m}$ within the coverage area. Table I summarizes the system parameters.

We assume a fixed network topology, and vary the parameters that are related to the wave activity (wave height and wave length). The surface parameters are taken from historical and prediction values from National Geophysical Data Center databases [11], [12]. We also account for the fact that an acoustic communication signal does not consist of a single frequency, but occupies a (possibly wide) bandwidth. The overall transmission loss is thus computed over the entire frequency range, which is taken here to be $5 \mathrm{kHz}-15 \mathrm{kHz}$.

Each execution of the Bellhop tool [7] takes about 5 minutes on an Intel Core ${ }^{\mathrm{TM}} 2 \mathrm{Duo}$ CPU $2.10 \mathrm{GHz}$ processor running on a standard laptop computer with $3 \mathrm{~GB}$ of RAM memory. Considering 14 different wave heights and 14 different wave lengths, i.e. 196 different scenarios, the complete analysis lasts about 16 hours for a single source location and a single frequency within the signal bandwidth.

Each simulation run produces the acoustic field values in a $5 \mathrm{~km} \times 5 \mathrm{~km} \times 30 \mathrm{~m}$ volume, with a resolution of $0.33 \mathrm{~m}^{3}$. The values corresponding to selected receiving node locations are then extracted, and a statistical analysis is performed for each location.

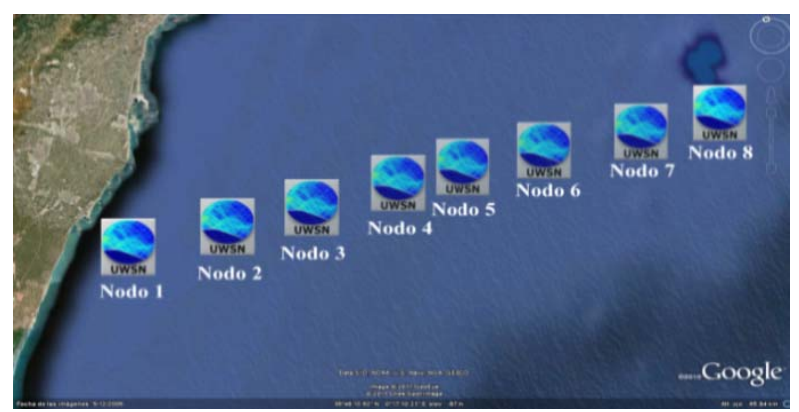

Figure 2. Network deployment in Valencia, Spain.

TABLE I

SYSTEM PARAMETERS

\begin{tabular}{|c|c|}
\hline transmission range & $500 \mathrm{~m}$ to $3700 \mathrm{~m}$ (in steps of $\sim 500 \mathrm{~m}$ ) \\
\hline area & $5000 \mathrm{~m} \mathrm{x} 5000 \mathrm{~m}$ \\
\hline sediment floor & Gravel \\
\hline frequency & August \\
\hline Month & $15 \mathrm{kHz}$ \\
\hline wave height & $100 \mathrm{~m}$ to $150 \mathrm{~m}$ (in steps of $3.5 \mathrm{~m}$ ) \\
\hline wave length & $25 \mathrm{~m}$ to $35 \mathrm{~m}$ \\
\hline water depth & $10 \mathrm{~m}$ \\
\hline system depth &
\end{tabular}

\section{Statistical Propagation Model}

The statistical propagation model is built by compiling the transmission loss values obtained from the deterministic model. The values of transmission loss, expressed in $\mathrm{dB}$ (logarithmic scale) are treated as random variables, and it is implicitly assumed that all surface conditions are equally likely.

Fig. 3 shows the histogram of the values obtained for Node 2, which is $500 \mathrm{~m}$ away from the source, and Fig. 4 shows the histogram obtained for Node 4, which is $1600 \mathrm{~m}$ away from the source. Shown also in these figures is a normal distribution with mean and variance equal to the ensemble averages of the transmission loss (solid curves).

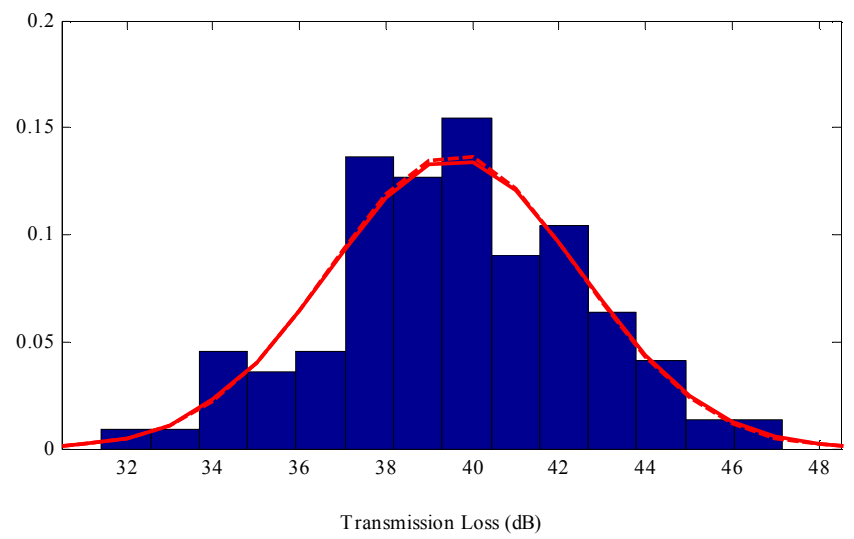

Figure 3.Histogram of the transmission loss calculated for Node 2 using the deterministic propagation model for varying surface conditions. 


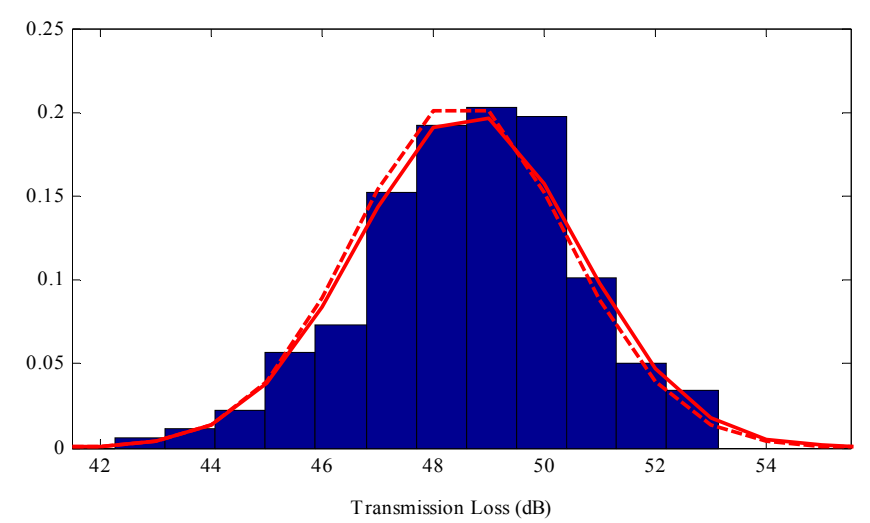

Figure 4.Histogram of the transmission loss calculated for Node 4 using the deterministic propagation model for varying surface conditions.

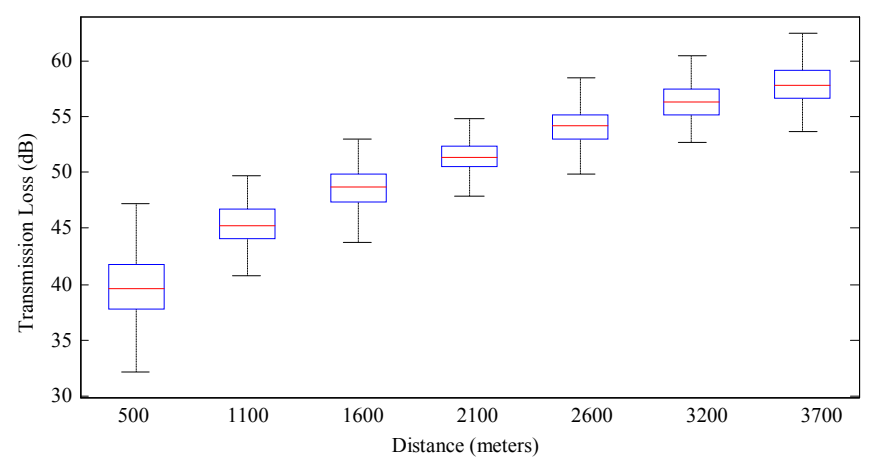

Figure 5. Transmission loss mean value and standard deviation (boxed) versus distance.

The mean and variance obtained for different ranges from the source (different locations of the receiving node) are shown in Fig.5. We note that the mean value of transmission loss increases with distance, as dictated by the energy spreading. The variance, however, does not change as much with the distance. This fact motivates us to assume the same variance for a range of distances. Namely, we take the standard deviation to be $2.9 \mathrm{~dB}$ for node separations below 1 $\mathrm{km}$ (Node 2) and $1.9 \mathrm{~dB}$ for node separations between $1 \mathrm{~km}$ and $4 \mathrm{~km}$ (Nodes 3-8) in the present scenario.

A statistical model is now assumed, which generates the transmission loss as a normally distributed random variable on a logarithmic scale (or equivalently, log-normally distributed on a linear scale). The mean value for this model can be taken as the ensemble average obtained from the deterministic model for a given distance. However, another approach is possible as well, whose goal is to further reduce the computational demands involved in building the statistical model for a given deployment geometry. Namely, we assume that the mean value increases linearly with the logarithm of distance, and fit the measured (ensemble-averaged) values to specify the linear dependence. Fitting can also be performed individually for different ranges of distances, yielding the corresponding linear dependencies. In our example, this approach provided the mean of $-6.5 \mathrm{~dB}+1.7 \cdot 10 \mathrm{log}$ (distance $[\mathrm{m}]$ ) for shorter distances (Nodes 2-3) and -35.5 dB + 2.6.10 $\log ($ distance $[\mathrm{m}])$ for longer distances (Nodes 4-8).
TABLE II

TRANSMISSION LOSS PARAMETERS OBTAINED FROM THE DETERMINISTIC Propagation MODEl AND Statistical Propagation MODEL.

\begin{tabular}{|c|c|c|c|c|c|}
\hline \multirow{2}{*}{ Node } & \multirow{2}{*}{$\begin{array}{c}\text { Distance } \\
{[\mathrm{m}]}\end{array}$} & \multicolumn{2}{|c|}{ DPM } & \multicolumn{2}{c|}{ SPM } \\
\cline { 3 - 5 } & & mean [dB] & $\sigma[\mathrm{dB}]$ & mean $[\mathrm{dB}]$ & $\sigma[\mathrm{dB}]$ \\
\hline 2 & 500 & 39.58 & 2.95 & 39.56 & 2.9 \\
\hline 3 & 1,100 & 45.42 & 2.07 & 45.40 & \\
\hline 4 & 1,600 & 48.62 & 1.99 & 48.49 & \multirow{2}{*}{1.9} \\
\hline 5 & 2,100 & 51.31 & 1.84 & 51.59 & \\
\hline 6 & 2,600 & 54.21 & 1.97 & 54.02 & \\
\hline 7 & 3,200 & 56.48 & 1.87 & 56.39 & \\
\hline 8 & 3,700 & 57.99 & 1.76 & 58.04 & \\
\hline
\end{tabular}

TABLE III

KULLBACK-LEIBLER DisTANCE.

\begin{tabular}{|c|c|c|}
\hline Node & Distance $[\mathrm{m}]$ & KL distance \\
\hline 2 & 500 & 0.039 \\
\hline 3 & 1,100 & 0.028 \\
\hline 4 & 1,600 & 0.023 \\
\hline 5 & 2,100 & 0.021 \\
\hline 6 & 2,600 & 0.018 \\
\hline 7 & 3,200 & 0.015 \\
\hline 8 & 3,700 & 0.012 \\
\hline
\end{tabular}

Table II list the various values in question. We note that the nominal transmission loss (mean value listed for SPM) differs very little from the value calculated by ensemble averaging of the deterministic model's outputs (mean value listed for DPM). The pdf resulting from the statistical model is shown in Figs.3 and 4 as a dashed curve.

We note that the distributions resulting from the two models are quite similar. In order to quantitatively judge the validity of the hypothesized statistical model, we have calculated the Kullback-Leibler (KL) distance [13] between the pdf estimated from the deterministic model (histogram of Fig.3 and 4) and the Gaussian pdf used for the statistical model. This distance is zero when the two distributions are identical. In Table III, we list the KL distance for every sourcedestination pair considered.

\section{IMPLICATIONS FOR NETWORK PLANNING}

The apparent match between the results of deterministic and statistical models motivates the use of SPM for network design and analysis via simulation. Consider, for example, network simulation over a prolonged interval of time that spans varying propagation conditions and involves transmission of a large number of data packets over multiple hops. If deterministic modeling is used, each packet transmission requires one execution of the Bellhop ray tracer, which soon becomes excessively long for a growing number of data packets (assuming 5 minutes for each Bellhop run and a single frequency, 100,000 packets would take about a year.) Although the DPM offers an exact solution for the particular geometry observed at any given moment in time, its execution 
makes the simulation times unaffordable for benchmarking and testing of the upper layer protocols.

In contrast, a statistical model can take several hours to compute (16 hours in the example we presented), but once computed, each realization requires only a single call to a Gaussian random number generator. Moreover, if the network topology changes slightly, or if a new node is added, the statistical model needs to be augmented only by the corresponding set of nominal parameters (mean and standard deviation for the newly created links.

Most importantly, the statistical model can easily be used to assess transmit power allocation that will guarantee successful data packet reception with a desired level of performance (e.g. link reliability). Namely, the SPM can easily be used to calculate the transmission loss values that are not exceeded with a given probability. For example, a $90 \%$ transmission loss is that value which is not exceeded for $90 \%$ of time, i.e. in $90 \%$ of channel realizations. Fig. 6 shows the $50 \%$ and $90 \%$ transmission loss for our example system. We observe a good match between the values predicted by the deterministic model, and those of the statistical model. Note that the X\% values of the SPM are computed analytically, based only on the knowledge of the mean and standard deviation.

The availability of $\mathrm{X} \%$ values is significant for determining the transmit power necessary to achieve a certain level of performance. Typically, network planning is based on the nominal ray trace, i.e. on the $50 \%$ transmission loss to which some margin may be added. If transmit power allocation is based on a different value, say $90 \%$ transmission loss instead of the nominal $50 \%$, data packets will be more likely to reach their destinations. More power will be needed at the same time, but the overall network performance may improve. We say may improve, because a higher transmit power also implies higher levels of interference. The resulting performance tradeoffs are generally hard to address analytically, and are instead assessed via simulation. A statistical propagation model that directly links the transmit power to the $\mathrm{X} \%$ transmission loss then becomes a meaningful and useful tool for system design.

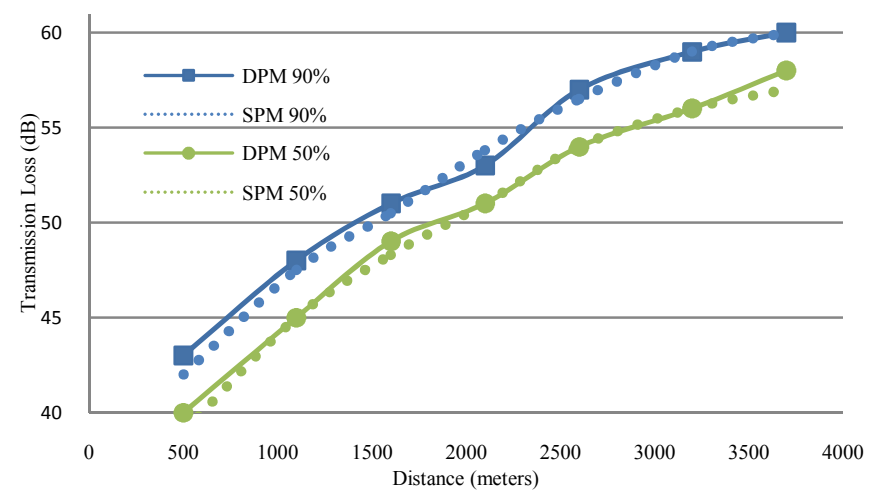

Figure 6. Transmission loss value that is not exceeded with a given probability $(50 \%, 90 \%)$ is shown versus distance. The solid and dashed curves show the results obtained from the deterministic and the statistical propagation models, respectively.

\section{CONCLUSIONS}

Large-scale design of an underwater acoustic network requires a judicious allocation of the transmit power across different links to ensure a desired level of system performance (connectivity, throughput, reliability, etc.). Because of the inherent system complexity, simulation analyses are normally conducted to assess the performance of candidate protocols under different resource allocation policies. These analyses are often restricted to using deterministic propagation models, which, although accurate, do not reflect the randomly timevarying nature of the channel.

While it is possible in principle to examine the network performance for a large set of perturbed propagation conditions, the computational complexity involved in doing so is extremely high. To facilitate network simulation in the presence of channel fading, we investigated a statistical modeling approach. Our approach is based on establishing the nominal system parameters for a desired deployment location (water depth, sediment composition, operational frequency range) and using ray tracing to compute an ensemble of transmission losses for typical inter-node distances. An ensemble is generated by considering a set of perturbed surface conditions, defined by varying wave activity (height, period). The so-obtained ensemble is then used to determine the statistical parameters of a hypothesized log-normal distribution of the transmission loss. For a representative example of a small network operating in a $5 \mathrm{~km} \times 5 \mathrm{~km}$ area with inter-node distances ranging between $500 \mathrm{~m}$ and $4 \mathrm{~km}$, it was found that the mean can be well approximated as a linear function of the logarithm of distance, while the variance can be modeled as constant over given ranges of distances. Models that are more elaborate and more accurate than the log-normal one can also be developed using this approach.

A statistical model of this type enables computationallyefficient inclusion of fading effects into a network simulator. Namely, to assess the average system performance, network operation has to be simulated over a large set of channel realizations (e.g. varying surface conditions). Whereas repeated computation of the ray trace for different hops that each of the data packets traverses in a given network may be computationally prohibitive, statistical modeling requires only a single call to the Gaussian random generator for each packet transmission. The overall simulation time is thus considerably reduced, allowing a system designer to freely experiment with varying protocols and resource allocation strategies in an efficient manner. The ultimate goal of such computational experiments is to choose the best upper-layer protocol suite and to relate the necessary system resources (power, bandwidth) to the propagation conditions, i.e. to the statistical parameters of the transmission loss (e.g. X\% value), which can in turn be easily generated using the proposed method of statistical modeling. 


\section{ACKNOWLEDGMENT}

This work was supported by the Ministry of Science and Education of Spain under grant DPI2007-66796-C03-03, and in part by the NSF grants 083172 and 0946610.

\section{REFERENCES}

[1] J.A. Rice, W.O. Che, "A Discovery Process for Initializing Underwater Acoustic Networks," in Proc. IEEE Sensorcomm, pp.408-415, July 2010.

[2] F. Guerra, P. Casari, M. Zorzi, "A performance comparison of MAC protocols for underwater networks using a realistic channel simulator," in Proc. IEEE Oceans Conf, pp.1-8, Oct. 2009.

[3] G. Xie, J. Gibson, L. Diaz-Gonzalez, "Incorporating Realistic Acoustic Propagation Models in Simulation of Underwater Acoustic Networks: A Statistical Approach," in Proc. IEEE Oceans Conf, pp.1-9, Sept. 2006.

[4] J. Llor, M. Malumbres, "Performance Evaluation of Underwater Wireless Sensor Networks with OPNET," in Proc. ACM Simutools. ICST, Barcelona, Spain, March 2011.
[5] B. Tomasi, P. Casari, L. Badia, M. Zorzi, "A Study of Incremental Redundancy Hybrid ARQ over Markov Channel Models Derived from Experimental Data," in Proc. ACM WUWNet, Woods Hole, MA, Sep. 2010.

[6] W.-B. Yang, T.C. Yang, "Characterization and Modeling of Underwater Acoustic Communications Channels for Frequency-Shift-Keying Signals," in Proc. IEEE Oceans Conf, pp.1-6, Sept. 2006.

[7] M. Porter et al., "Bellhop code." [Online]. Available: http://oalib. hlsresearch.com/ Rays/index.html.

[8] "National geophysical data center, seafloor surficial sediment descriptions". [Online]. Available: http://www.ngdc.noaa.gov/mgg/ geology/deck 41.html.

[9] "General bathymetric chart of the oceans". [Online]. Available: http:// www.gebco.net.

[10] "World ocean atlas". [Online]. Available: http://www.nodc.noaa.gov/ OC5/WOA05/pr_woa05.html.

[11] "METEOSIM", [Online]. Available: http://www.meteosim.com.

[12] Puertos del Estado, [Online]. Available: http://www.puertos.es.

[13] A. Rényi, Probability Theory. New York: Elsevier, 1970. 